\title{
Anesthesia for flap surgery in a patient with polymyositis
}

\author{
Sowoon Ahn ${ }^{1}$, Ju Ho Lee ${ }^{1}$, Eun Ah Yang ${ }^{1}$, Chunghyun Park ${ }^{1}$, and Dong Wook Shin ${ }^{2}$ \\ Department of Anesthesiology and Pain Medicine, ${ }^{1} \mathrm{CHA}$ Bundang Medical Center, CHA University, Seongnam, ${ }^{2} \mathrm{CHA}$ Gumi Medical \\ Center, CHA University, Gumi, Korea
}

Polymyositis is a subacute inflammatory myopathy presenting as progressive and often symmetric muscle weakness. It is believed that the patients with polymyositis are sensitive to nondepolarizing muscle relaxants. The major concerns for the anesthesiologist are delayed recovery from muscle relaxation, aspiration pneumonitis, arrhythmias and cardiac failures [1].

A 26-year-old male with polymyositis, $170 \mathrm{~cm}$ in height and $76 \mathrm{~kg}$ in weight, had flap surgery of sacral pressure sore which developed during intensive care. He had progressive muscle weakness for one year. Three months ago, he was admitted to a hospital under the impression of viral hepatitis or nonalcoholic steatohepatitis due to elevated liver enzymes. However, viral studies and liver biopsy results were normal. Instead, elevated $\mathrm{CK}$ (> 11,000 U/L) and CK-MB (> $300 \mathrm{ng} / \mathrm{ml}$ ) were found. Polymyositis was diagnosed according to clinical features, electromyography results and muscle biopsy findings. During the hospital stay, he presented pneumonia symptoms. Intravenous antibiotics were administered, but the patient was not improved. Three days later, dyspnea became more severe and arterial blood gas analysis (ABGA) showed $\mathrm{PaO}_{2} 49 \mathrm{mmHg}$ and $\mathrm{SaO}_{2} 80 \%$. Upon the diagnosis of acute respiratory distress syndrome, the patient was intubated. Despite of ventilator care, pulmonary status deteriorated and extracorporeal membrane oxygenation (ECMO) support was applied for five days. His pulmonary function improved enough to switch to conventional ventilator care and the care was maintained for another ten days. He was transferred to general ward after recovery of pulmonary function.

On the day of surgery, the patent's laboratory findings, chest $\mathrm{X}$-ray and vital signs showed normal. In the operating room, direct arterial pressure, electrocardiography, end-tidal $\mathrm{CO}_{2}$, tem- perature, pulse oxymetry and peripheral nerve stimulator (TOFWatch, Organon Inc., Dublin, Ireland) were monitored. Neuromuscular relaxation was assessed by train of four (TOF) at the adductor pollicis with supramaximal stimulation of the ulnar nerve at $2 \mathrm{~Hz}$ every 12 seconds and was recorded continuously until end of anesthesia. Anesthesia was induced with glycopyrrolate $0.2 \mathrm{mg}$, propofol $120 \mathrm{mg}$ and remifentanil infusion. The lungs were ventilated with $100 \%$ oxygen and $2.5 \%$ sevoflurane. TOF-ratio was assessed before administration of muscle relaxant as a reference value and was recorded continuously until end of anesthesia. Rocuronium $10 \mathrm{mg}$ IV was slowly started and TOF-ratio decreased to $50 \%$ of reference value after 5 minutes. Rocuronium $10 \mathrm{mg}$ was added and TOF-ratio was suppressed to 0 after another 5 minutes. The patient was presenting excellent intubating conditions according to the Copenhagen Consensus Conference score (abducted vocal cords, no vocal cords movement, no coughing, no limb movement). Anesthesia was maintained with $1.5 \mathrm{~L} / \mathrm{min}_{2}, 1.5 \mathrm{~L} / \mathrm{min}$ air, sevoflurane and remifentanil. Operation time was 250 minutes. TOF-ratio maintained 0 by 110 minutes after induction. Then TOF-ratio started to increase slowly but maintained $<50 \%$ by 200 minutes and increased to $75 \%$ at the end of the surgery. The surgery did not need intensive relaxation and patient did not have spontaneous respiration; therefore, no additional rocuronium was given. ABGA revealed adequate oxygenation and spontaneous respiration was restored after switching to manual ventilation. Ten mg of pyridostigmine and $0.2 \mathrm{mg}$ of glycopyrrolate were injected and TOF-ratio increased $>90 \%$ after 5 minutes. He was extubated and transferred to recovery room. There was no specific complication during the hospital stay.

Corresponding author: Dong Wook Shin, M.D., Department of Anesthesiology and Pain Medicine, CHA Gumi Medical Center, CHA University, 12, Sinsi-ro 10-gil, Gumi 463-712, Korea. Tel: 82-54-450-9691, Fax: 82-54-450-9990, E-mail: for7518@naver.com

(c) This is an open-access article distributed under the terms of the Creative Commons Attribution Non-Commercial License (http:// creativecommons.org/licenses/by-nc/3.0/), which permits unrestricted non-commercial use, distribution, and reproduction in any medium, provided the original work is properly cited. 
Polymyositis is a rare disease with the prevalence from 2.410.7 cases per 100,000 persons. The principal feature is muscle weakness, which may be generalized, but it most commonly presents with bilateral involvement of the pelvis, shoulder and neck muscles [1]. The associated extra muscular manifestations include systemic symptoms such as fever, malaise, weight loss, arthralgia, Raynaud's phenomenon, cardiac disturbances including atrioventricular conduction defects, tachyarrythmias, dilated cardiomyopathy and a low ejection fraction, pulmonary dysfunction due to weakness of thoracic muscles and interstitial lung disease [1]. In this case, the patient had chronic muscle weakness and an acute respiratory distress which required ECMO treatment. The patient's muscle biopsy findings showed fiber necrosis, atrophy and multifocal inflammatory cell infiltrations competed with inflammatory myopathy. The patient's EMG findings revealed severe denervation potentials on multiple muscles including limb muscles. Anesthetic considerations in polymyositis are respiratory insufficiency, aspiration pneumonia, arrhythmias, cardiac failure and hyperkalemia [2]. It is believed that the patients with polymyositis are sensitive to nondepolarizing muscle relaxants, and the use of their antagonist drugs may cause muscle weakness and severe dysrhythmias [2]. Ueki et al. [3] suggested that the anesthetist should be careful when using muscle relaxants on those patients. The use of neuromuscular blocking agents in patients with neuromuscular disease is controversial because of its prolonged duration of ac- tion. Neuromuscular blockade monitoring using a peripheral nerve stimulator is suggested due to lack of standard recommendations regarding the application of nondepolarizing muscle relaxants [3]. Ohta et al. [4] used no muscle relaxants for endotracheal intubation of two polymyositis patients and maintenance of their anesthesia. In this case, the patient was intubated with rocuronium $20 \mathrm{mg}(0.26 \mathrm{mg} / \mathrm{kg})$, which was less than half of usual intubating dose, and maintained without additional muscle relaxant. In polymyositis, intramuscular blood vessels show endothelial hyperplasia, fibrin thrombi, and obliteration of capillaries, resulting in a reduction in capillary blood flow to the muscles. Therefore, the slower delivery of rocuronium to the neuromuscular junction may result in slower onset of action and long reversal time of rocuronium [5]. We were unable to affirm the slow onset of rocuronium in this case because small additive doses of rocuronium were injected. Considering the first $10 \mathrm{mg}$ of rocuronium as a preceding dose, 5 minutes after the second injection would be defined as the onset time because T1 disappeared in 5 minutes. However, such a small dose was enough to intubate the patient and extend the duration of muscle relaxation to an unusual manner. Even though the patient experienced no problem during the recovery, if the surgery time was shorter, his recovery would have been delayed. In conclusion, titrated small dose of muscle relaxant in conjunction with a peripheral neuromuscular monitor is a safe option for the polymyositis patient where the use of neuromuscular blockers is unavoidable.

\section{References}

1. Harrison TR. Harrison's Principles of Internal Medicine, 17th ed. New York, McGraw Hill. 2008, pp 2696-700.

2. Garg R, Bhalotra AR, Bhadoria P, Anand R, Kumar A. Muscle disorder-experience with two rare cases. J Anaesth Clin Pharmacol 2008; 24: 225-8.

3. Ueki M, Tosaki Y, Ogli K, Uefuji T. Anesthetic management of a patient with dermatomyositis--clinical observation of the effect of muscle relaxants. Masui 1989; 38: 1505-8.

4. Ohta M, Nishikawa N, Kida H, Miyao S. Anesthetic management of two patients with polymyositis. Masui 2000; 49: 1371-3.

5. Suzuki T, Kitajima O, Ueda K, Kondo Y, Kato J, Ogawa S. Reversibility of rocuronium-induced profound neuromuscular block with sugammadex in younger and older patients. Br J Anaesth 2011; 106: 823-6. 